\title{
Precise determination of the Poisson ratio in soft materials with 2D digital image correlation
}

\author{
Robyn H. Pritchard, Pascal Lava, Dimitri Debruyne, Eugene M. Terentjev \\ Cavendish Laboratory, University of Cambridge, JJ Thomson Avenue, Cambridge CB3 OHE, U.K. \\ Department of Mechanical Engineering, Catholic University College Ghent, Gebroeders De Smetstraat 1, B-9000 Ghent, \\ Belgium \\ Department MTM, Katholieke Universiteit Leuven, Kasteelpark Arenberg 44, B-3001 Leuven (Heverlee), Belgium
}

\begin{abstract}
In this article, we demonstrate the application of digital image correlation (DIC) in evaluating the strains and Poisson ratio of a range of soft materials in terms of their spatial and temporal resolutions. Four samples of Polydimethylsiloxane (PDMS) were used as control substances and were measured to have Poisson ratios of $0.498,0.503,0.500$, and 0.499 , in agreement with the reported incompressible value of 0.50 [1]. Two carbon nanotube (CNT) elastomers of identical composition, but one of a homogeneous and the other of an inhomogeneous CNT distribution, were used to determine the spatial resolution with good results (Fig. 2 and Fig. 4). The relaxation of a 3D polydomain liquid crystal elastomer (3D-LCE), a cholesteric liquid crystal elastomer (CLCE), and a polyacrylamide gel (PAAm) in water, were used to determine the temporal resolution. A $10 \mathrm{~min}$ video short at $25 \mathrm{fps}$ was used to evaluate the time dependence of the 3D-LCE over which time an increase in the Poisson ratio was observed. The 3D-LCE relaxes from its initial state at 0.42 to 0.50 , converging towards incompressibility. The CLCE was found to have a similar initial value of 0.44 but converged to $\sim 0.60$, a consequence of its anisotropic nature. PAAm gel relaxation in water was studied over a time period of 7 hours with digital images taken periodically every minute. Its Poisson ratio was found to decrease smoothly from 0.50 to 0.26 , with an accompanying reduction in force. The equilibrium result compares well to the 0.25 value predicted by theories of the strain induced swelling of dilute gels. In summary, we find DIC to be a powerful and easy to implement method of accurately measuring local strains in a range of soft materials.
\end{abstract}

\section{Introduction}

In linear viscoelasticity, the Poisson ratio is a particularly important yet difficult to measure quantity. Defined as the ratio of transverse strain to parallel strain for a small uniaxial extension, it describes the volume response to deformation. Although it is often reported as a constant, it is infact a time dependent quantity and little work has been done in determining its viscoelastic nature 2 , 3. With the increase in computational modelling of materials and composites, a precise knowledge of viscoelastic constants is needed to ensure their accuracy. This is particularly important for more complex materials such as composites and other systems with microstructure, notably biological materials, which may not deform homogeneously.

In soft materials, a non-contact measurement of strain is preferable to contact methods such as clip on or contact extensiometers, as they can lead to unreliable results; the weight of the device, as well as the point of contact at clamping, may strongly influence any measurements made 4. In this paper, we utilise the academic digital image correlation (DIC) software, MatchID, as a contactless method for full field strain analysis (http://www.matchid.org). This offers a distinct advantage compared to video extensiometry,

Email address: emt1000@cam.ac.uk (Eugene M. Terentjev) 
which measures the strain between two marked edges and therefore only measures the average strain. Digital image correlation, on the other hand, divides a patterned material surface into identifiable subsections and tracks their displacement from an initial image before deformation occurs to successively analysed images post deformation. Local strains can then be calculated from displacements points to a high degree of accuracy 5 .

DIC was first developed by researchers at the University of South Carolina in the early 1980s [6], and since then has seen many advances. For a thorough discussion of the history of digital image correlation, as well as its techniques, see [7. With advancements in computer and image technology, as well as reduced hardware cost, DIC is becoming more accessible [8. In recent years, this technique has been used to characterise a range of materials including polymers [4, 9], foam [10], textiles [11], and biological materials, such as the human ear drum [12] and mouse arteries 13$]$.

In this work, we demonstrate the applications of DIC in characterising the Poisson ratio through uniaxial relaxation tests on a range of polymeric and composite materials; namely, polydimethylsiloxane (PDMS), two carbon nanotube (CNT) elastomers, 3D polydomain liquid crystal elastomer (3D-LCE), cholesteric liquid crystal elastomer (CLCE), and a polyacrylamide (PAAm) gel immersed in water. We emphasise two aspects of DIC: its ability to map local strains to a high degree of resolution, and the large range of experimental timescales.

Spatial resolution is determined by comparing two carbon nanotube elastomers of $2 \%$ weight CNTs. The distribution of CNTs in the polymer matrix is determined in preparation by the extent of mechanical mixing. Short mixing times give an inhomogeneous composite with localised clusters of CNTs, whereas long times give a homogeneous composite of evenly distributed CNTs [14.

The time response at the (sub second) scale was tested using two liquid crystal elastomers (LCEs). LCEs are unique in that they combine rubber like elasticity with the symmetry breaking and optical properties of liquid crystals. Their irregular behaviour arises from the presence of anisotropic rigid units, either in the main polymer chain or as side chain units, known as mesogens [15]. The ordering of the mesogenic units give rise to liquid crystalline like phases. 3D-LCEs consist of micrometre-sized nematically ordered domains and are locally anisotropic [16. However, on the macroscopic scale, the directors of all domains cancel and the material possesses no macroscopic anisotropy. In CLCEs mesogens are aligned in offset nematic planes forming a repeating helical structure. Under strain, the material behaves anisotropically as a result of the preferential realignment of directors to stretch [17]. In general, the realignment of mesogens will give a soft elastic response at long times (low frequencies), but at short times (high frequencies) an order of two magnitude increase in the shear modulus has been reported [16. The large changes in shear modulus over measurable time scales, as well as their potential anisotropic behaviour, make them valuable subjects for measuring the time dependent Poisson ratio, which to the author's knowledge has not yet been reported for LCEs.

Long time scales were examined with relaxation experiments on a polyacrylamide gel immersed in water. It is known that free swollen gels will further take in solvent under extensive strain and expel solvent under compressive strain 18. The change in Poisson ratio is dominated by the diffusion rate of solvent into the gel and for PAAm samples with thickness on the mm scale, equilibrium is reached over a period of hours 19 .

In this article, we use the Henky strain tensor [20 to describe strain, defined as $\ln \left(\lambda_{i}\right)=\ln \left(1+\varepsilon_{i}\right)$, where $\lambda_{i}$ and $\varepsilon_{i}$ are the stretch ratio and engineering strain in the $i^{t h}$ direction, respectively. The Poisson ratio calculated with the Hencky strain is a constant for incompressible materials, whereas for other strain determinations it will vary with imposed strain [4]. For an isotropic incompressible material $\lambda_{\|} \lambda_{\perp}^{2}=1$ and therefore $\nu=-\ln \left(\lambda_{\perp}\right) / \ln \left(\lambda_{\|}\right)=-\ln \left(\lambda_{\|}^{-0.5}\right) / \ln \left(\lambda_{\|}\right)=0.5$., where $\lambda_{\|}$and $\ln \left(\lambda_{\perp}\right.$ are the parallel and transverse stretch ratios, respectively (see Fig. 1). 


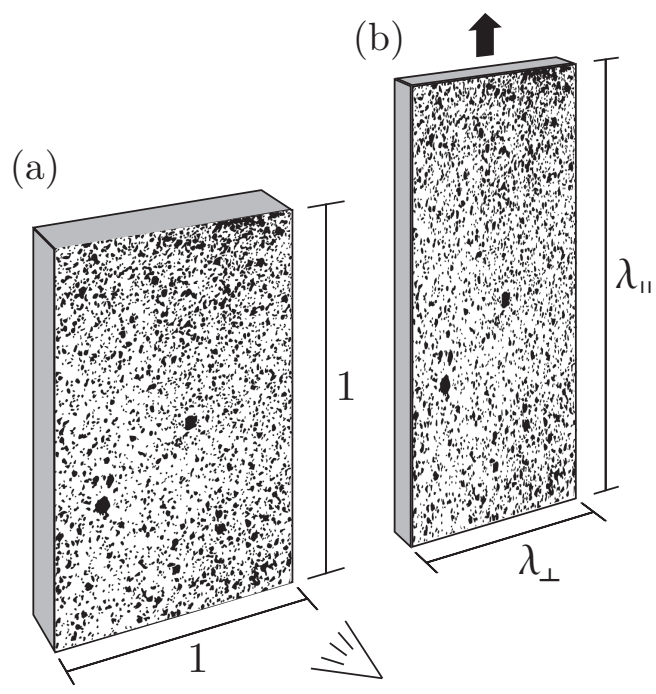

Figure 1: (a) shows the sample before stretch of unit dimensions of 1, a speckle pattern is applied with paint. (b) shows the sample after $\lambda_{\|}$stretch ratio applied, with $\lambda_{\perp}$ strain ratio response in the free direction. The speckle pattern deforms with the material surface.

\section{Experimental Section}

\subsection{Materials}

Polydimethylsiloxane (PDMS): A Dow Corning Sylgard 184 PDMS elastomer kit was used as received. The curing agent and prepolymer were thoroughly mixed at a 1:10 weight ratio and degassed for an hour. The mixture was then sealed in an acrylic mold and left in a heat oven for 6 hours at 60 degrees to set. Upon setting, 5 samples of dimensions $22 \mathrm{~mm} \times 12 \mathrm{~mm} \times 1.5 \mathrm{~mm}$ were removed from the mould.

Carbon nanotube elastomers: With the use of specially designed surfactant molecules [21] in order to ensure full solubility, we were able to homogeneously disperse multi-walled carbon nanotubes (CNTs) in PDMS matrix, and then crosslink it to fix their configuration. Although a growing number of applications use CNT/PDMS composites, the issues associated with CNT dispersion persist and in many cases the nanotubes remain in small bundles and clusters. The use of special surfactants requires a much smaller quantity of solvent during the solution processing: for example, pure PDMS melt (Dow Corning Sylgard 184) could be directly dissolved into the sonicated surfactant/CNT-PET solution at a 1:4 PDMS to PET to produce a homogeneous composite with single-nanotube level of dispersion after 5 hours of shear mixing. For comparison, we also used a deliberately poorly dispersed CNT/PDMS composite, which was only mechanically mixed for 10 minutes.

Polyacrylamide gel: PAAm gels were prepared by radical copolymerisation of a stock solution of $30 \%$ wt Acrylamide (AA) and $N, N$ '- methylenebis-acrylamide (BIS) at a ratio of 37.5:1.0 Ammonium persulfate (APS) was used as the initiator and $N, N, N^{\prime}, N^{\prime}$ - tetramethylethylenediamine (TMED) as an accelerant. Gelation was conducted at room temperature for 1 hour in a sealed, Teflon coated, acrylic mould giving 4 uniform gel strips of dimensions $22 \mathrm{~mm} \times 12 \mathrm{~mm} \times 1.5 \mathrm{~mm}$. After gelation, the strips were gently removed and then submerged in a reservoir of distilled, deionised water. The gels were left for 48 hours before testing, and the water was changed regularly to remove any residual reactants.

Polydomain liquid crystal elastomer: Details of the synthesis of the siloxane-based nematic liquid crystalline elastomers can be found in 22. Our aim was to obtain a true polydomain state of the nematic phase, which can only be generated when the crosslinking is fully random with no internal stresses, and entanglements are frozen into the resulting network. In order to achieve such a configuration, we used a toluene solvent and completed the crosslinking reaction in the highly swollen isotropic phase of the polymer. After the reaction, the samples were slowly deswollen so that the dry elastomer network would not end up 
overentangled. For our purposes, this forms a perfectly isotropic elastic material which (unusually) has an extremely high mechanical loss factor [23] and an extremely long time stress relaxation [24].

Cholesteric liquid crystal elastomer: Synthesis was carried out following the general method introduced by Kim and Finkelmann [25] by substituting 25\% of the mesogenic side-groups with a chiral derivative of cholesterol pentenoate. Siloxane backbone chains were reacted under centrifugation with the mesogenic sidegroups and the crosslinker for 45 minutes to form a partially cross-linked gel. The reactor was then opened and the crosslinking reaction proceeded for the further 4 hours, during which time the solvent evaporated, leading to an anisotropic deswelling of the gel. All of the volume change in this setup occurs by reducing the thickness of the gel, while keeping the lateral dimensions fixed due to centrifugation. This introduces a very strong effective biaxial extension in the plane. Upon crosslinking, the director is forced to remain in the plane of stretching and results in the uniform cholesteric texture. For our purposes, this is a perfect transversely-isotropic elastic material [17].

\subsection{Setup and Analysis}

Tensile tests were performed in a computer controlled uniaxial stretcher. The stretcher is modular and has the option to attach an environmental chamber, in which samples can be strained whilst immersed in a temperature controlled fluid. All strains were applied at a cross head speed of $7 \mathrm{~mm} / \mathrm{s}$ to approximately $10 \%$ strain. The 2D DIC system consists of Canon 550D DSLR camera fitted with a Canon 100 mm macro lens. All images were 8-bit. The camera was placed in a secure mount and carefully aligned to be in plane with the sample.

For the PDMS, 3D-LCE and CLCE elastomers, video footage was taken during stretch at 25 fps at 1080p for 10 minutes. In the CNT experiments, digital images were taken every minute after stretch at a resolution of $5184 \times 3456$ pixels $(18 \mathrm{MP})$.In the PAAm experiment, the gel was secured to the clamps with acrylic resin after which one surface had moisture removed with tissue paper to allow a speckle pattern to be applied. During this process, the gel was never exposed to air for more than 2 minutes. The gel was then immersed in water at $25^{\circ} \mathrm{C}$ and left to equilibrate for 6 hours before testing. Images were taken every minute after strain was applied.

The speckle patterns were applied to the PDMS, 3D-LCE, CLCE and PAAm elastomers with matt spray paint (Fig. 1). In the case of the CNT elastomers, an air brush was used with white matt paint to give the finer speckle pattern that was needed to fully resolve the inhomogeneity. As the setup used 2D digital image correlation, no camera calibration is required. This is especially useful for the PAAm experiment in which the gel is viewed through an acrylic window. As the window is in plane to the sample and camera, refraction has no effect on strain measurements.

Table. 1 shows the setup and DIC parameters for each experiment. The camera distance was chosen to maximise the size of the sample in the field of view whilst accommodating the speckle size pattern. The conversion of pixels to mm was done through measuring the number of pixels in a known length (the clamps were machined to $15 \mathrm{~mm}$ ). The field of view indicates the full size of the image taken, this is a conversion from $5184 \times 3456$ pixels ${ }^{2}$ for full photos, $1920 \times 1080$ pixels $^{2}$ for images extracted from video, and $2592 \times 1718$ pixels $^{2}$ for half images (as is the case for PAAm). The spatial resolution is determined from the distance between two independent measurement points. For the displacement this is given by the subset size and not the step size, as any subsets shifted by some step size will be calculating displacements from at least two regions which have already been assessed and are thus not independent measurements. The resolution is calculated from a DIC analysis of two images taken before deformation in which the displacements and strains should be zero. It is quoted as the standard deviation of values and indicates the minimum which a signal can be said to be detected over camera and environmental noise. The mean is also listed and this is typically expected to be below the standard deviation. However, in some of the cases below it be seen the displacement mean is slightly above the standard deviation. This can be attributed to the soft nature of the samples allowing them small movement in response to vibrations and air flow. The sub-pixel resolutions are typical of DIC and originate from the additional information of matching a known pattern to the image [26].

Strains are described by the Virtual strain gauge (VSG), which gives the average strain components over a specific area, just as a physical strain gauge would. The size of this area is determined by the strain window 
of $\mathrm{N} \times \mathrm{N}$ displacement points. The spatial resolution of the VSG is the distance between each independent measurement of strain and is determined by $(S W-1) * S T+S S$, where $S W$ is the strain window size, $S T$ is the step size, and $S S$ is the subset size. Resolutions are calculated in the same manner as for displacement.

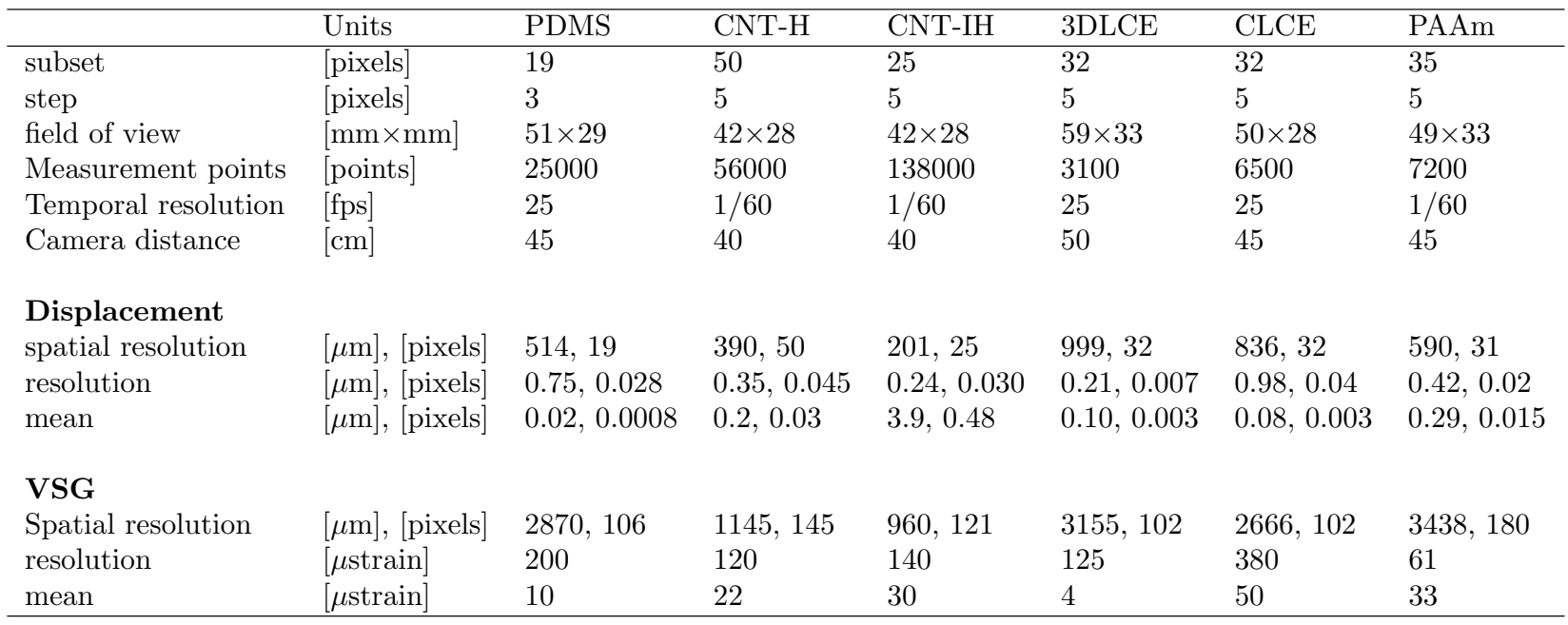

Table 1: Measurement information for the DIC tests presented in this paper. The Camera used was an 8-bit Canon 550 DSLR camera.

\section{Results}

\subsection{Resolving local deformation}

In this section, we examine the effectiveness of digital image correlation in resolving local inhomogeneities by comparing two carbon nanotube elastomers of identical composition but with different CNT distributions. The first elastomer was mechanically mixed for 10 minutes during preparation and has an inhomogeneous distribution of CNTs. The second was mixed for 5 hours to give a homogeneous distribution.

In Fig. 2 we show maps of the transverse strain $\left(E_{x x}\right)$, parallel strain $\left(E_{y y}\right)$, and Poisson ratio $(\nu)$ of the inhomogeneous CNT elastomers 10 minutes after application of strain. $E_{x x}$ and $E_{y y}$ are the Henky strains given by $\ln \left(\lambda_{x}\right)$ and $\ln \left(\lambda_{y}\right)$. The DIC analysis parameters are shown in Table. 1. In Fig. 2(a) and Fig. 2(b), which show the $E_{x x}$ and $E_{y y}$ strain maps, regions of localised deformation are clearly visible. A comparison of the maps reveals a good agreement: where there is small deformation in the transverse direction (red regions), there is a corresponding small deformation in the parallel direction (blue regions). The regions of small deformation are attributed to higher concentrations of CNTs as they increase the shear modulus [14. Conversely, regions of large deformation (blue in Fig. 2(a) and red in Fig. 2(b)) correspond to low concentrations of carbon nanotubes, where deformation is large. As expected for an inhomogeneous material, the spread of strains is large, with standard deviations of $\pm 20 \%$ and $\pm 19 \%$ for the $E_{x x}$ and $E_{y y}$ strains, respectively. A map of the Poisson ratio is shown in Fig 2(c), and although there are still large variations (standard deviation of $\pm 7 \%$ ), they are smaller than those seen in the strain maps. The narrower spread confirms the agreement of the $E_{x x}$ and $E_{y y}$ strain distributions and we find the average Poisson ratio to be 0.48 , close to a pure polymer value of 0.50 .

Although CNTs themselves are compressible with a Poisson ratio reported to be 0.29 [27, their shear modulus is order of magnitudes larger than the encapsulating polymer matrix 28]. At the low concentrations of CNTs tested, the bulk of the transverse contraction will be determined by the polymer response rather than that of the CNTs and a Poisson ratio of $\sim 0.50$ is to be expected. It should be noted that although the Poisson deviates from 0.50 locally, it does not mean there was compressible deformation, only that the deformation was anisotropic and that out of plane strain is not equivalent to the $E_{x x}$ strain (an assumption 
in the Poisson ratio calculation). Interestingly, these deviations average out when the mean is taken over the whole sample and incompressibility is almost entirely recovered.
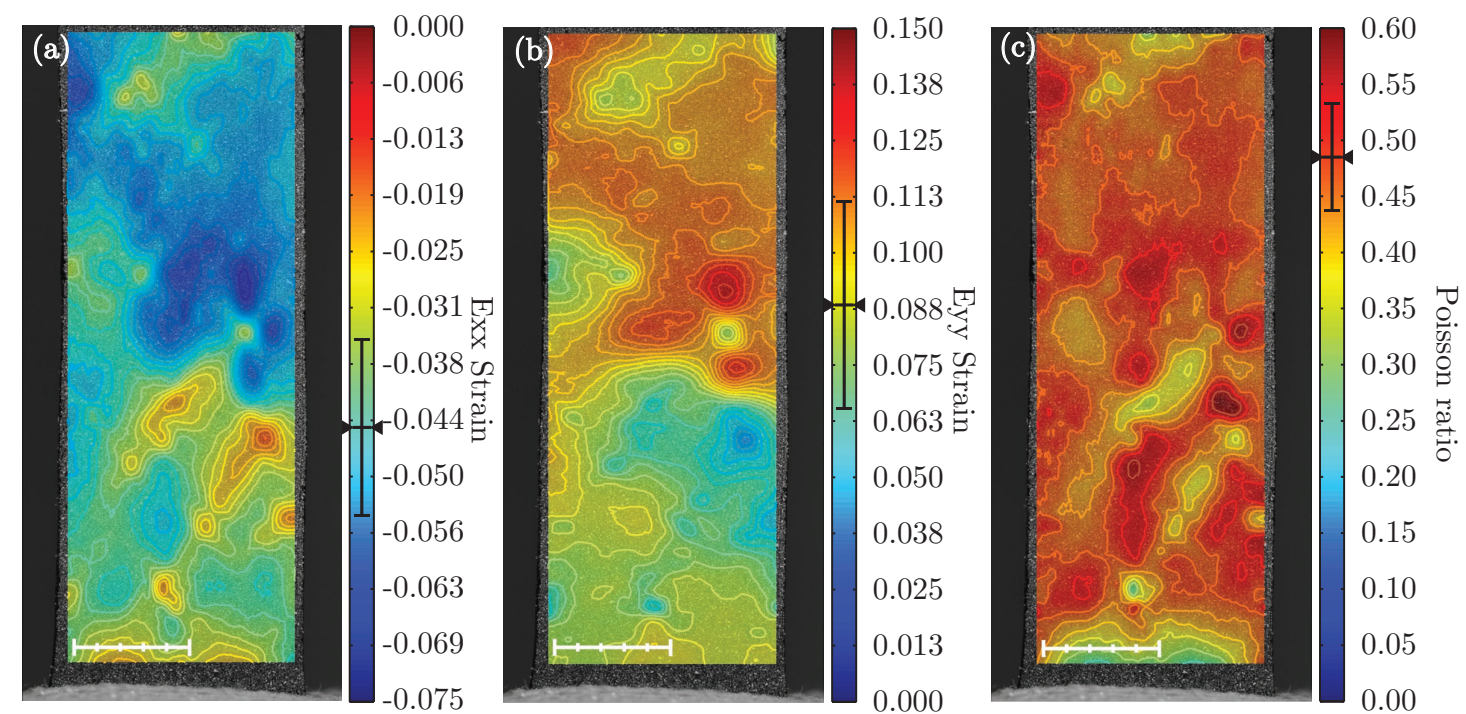

Figure 2: (a) $E_{x x}$ strain, (b) $E_{y y}$ strain, and (c) Poisson ratio maps of an inhomogeneous $2 \%$ wt carbon nanotube elastomer. The error bars embedded in each colour scale give the mean value as well as the corresponding standard deviation. The scale bar is $5 \mathrm{~mm}$.

In Fig. 3 maps of the $E_{x x}$ strain, $E_{y y}$ strain, and Poisson ratio of the homogeneous CNT elastomer 10 minutes after strain was applied are shown. The colour scales are identical to Fig. 2, In contrast to the inhomogeneous CNT elastomer, the maps show little variation, and the standard deviations of the $E_{x x}$ strain, $E_{y y}$ strain, and Poisson ratio are $\pm 2.0 \%, \pm 2.1 \%$, and $\pm 0.6 \%$, respectively. The mean value of the Poisson ratio is 0.495 , close to the incompressible value of pure polymer. With the DIC technique, the differences in homogeneity between the short time and long time mixed CNT elastomers, as well as the distribution of CNTs in the elastomer, are easily detectable. Although there are differences in the average Poisson ratio, the values are in a good agreement considering the large spear of values observed in the inhomogeneous CNT elastomer.
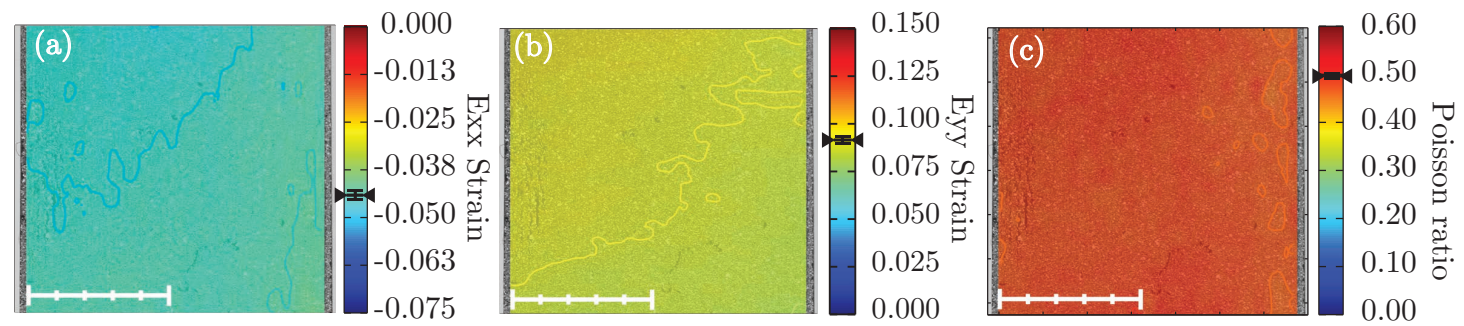

Figure 3: (a) $E_{x x}$ strain (b) $E_{y y}$ strain and (c) Poisson ratio maps of the homogeneous $2 \%$ wt carbon nanotube elastomer. The scales are equivalent to those in Fig. 2, and the scale bar is 5mm. The error bars embedded in each colour scale gives the mean value as well as its corresponding standard deviation.

The plots in Fig. 4 are the same as Fig. 3, but the scale limits are set between the minima and maxima of each map. On this scale, both strains appear to vary from a region of high deformation (in the top left) to a region of low deformation (in the bottom right). The smaller local fluctuations originate from camera noise 
as their variation is comparable to resolution listed in table. 1, this is not the case for the inhomogeneous CNT where local variations are at orders of magnitude larger than the resolution.

The origin of the large scale strain distribution is likely the result of three competing effects. (1) A genuine varying of the carbon nanotube distribution; however, this is unlikely to be so significant. (2) Misalignment of the sample, resulting in an applied strain that is not truly uniaxial; this is almost certainly occurring to some extent. (3) There is the possibility of offset or artificial strains as result of camera misalignment or out of plane motion. Out of plane motion typically refers to errors arising from deformation perpendicular to the image plane, resulting in an apparent change in size. These errors are minimised by an appropriate choice of lens and by placing the camera as far away from the sample as possible [7], as artificial strain errors go as $\Delta Z / Z$, where $Z$ is the camera distance and $\Delta Z$ is the change in distance upon stretch. In this experimental setup and over the large strains applied, uniform out of plane motion will have a minimal effect on strains. Nevertheless, soft materials present an additional problem in this respect as it is difficult to ensure the material deforms uniformly in the free directions, e.g., straightening of the material during stretch or curling at the edges, which may not be immediately obvious during experiment. Although the sample was not observably curved, there will undoubtedly be some level of nonuniform out of plane motion, which may affect results as sensitive as the Poisson ratio.

In this case, the strain distribution is unlikely to be result of out of plane motion or curvature, due to the agreement of $E_{x x}$ and $E_{y y}$ strains. If the out of plane motion does play a role, there would be a contrast between strains, e.g., curvature at the free edge would result in little change to $E_{x x}$ but decrease $E_{y y}$ [29]. As the strains are of physical origin, it is likely that the distribution is a result of sample misalignment resulting in non-uniform strain. It is important to note that all deformations will appear inhomogeneous on some scale, as errors will always manifest, it is only in comparison between samples that deformations can be termed homogeneous or inhomogeneous.
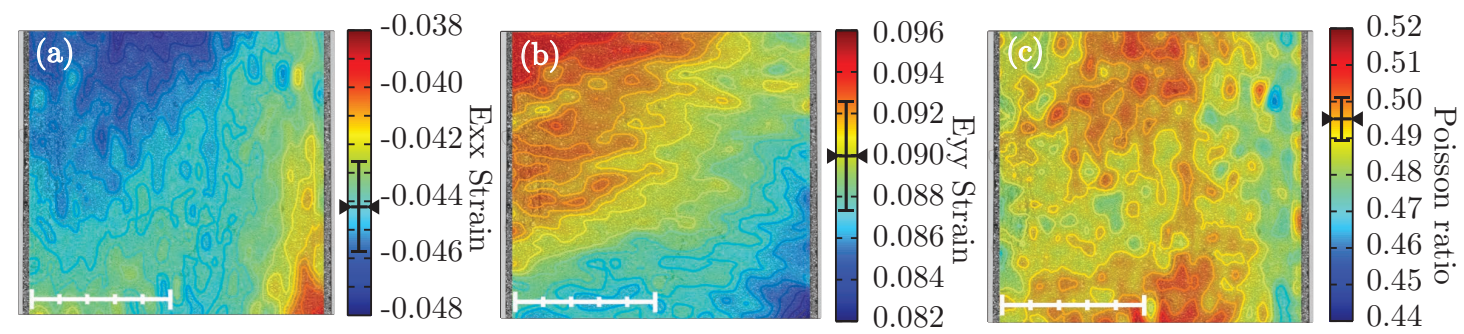

Figure 4: (a) $E_{x x}$ strain (b) $E_{y y}$ strain and (c) Poisson ratio maps of homogeneous $2 \%$ wt carbon nanotube elastomer. The error bars embedded in each colour scale give the mean value as well as the corresponding standard deviation. The scale bar is $5 \mathrm{~mm}$.

In Fig 5 relaxation plots of the normalised force and Poisson ratio are shown. The normalised force is taken as the current force value, $F(t)$, divided by the maximum post stretch value, $F_{\max }$. Both elastomers exhibit a long time viscoelastic force relaxation, due to a slow reconfiguration of the rigid CNT filler in the elastic matrix, with an equilibrium not having been reached in the experimental time span. In contrast, the Poisson ratio has no observable time dependence over the probed time scale. Here we plot the average Poisson ratio over the whole field of view of the film away from the clamps (see Discussion for details). The error bars indicate the Poisson ratio standard deviation over the analysed region and highlight the significant differences in homogeneity. 


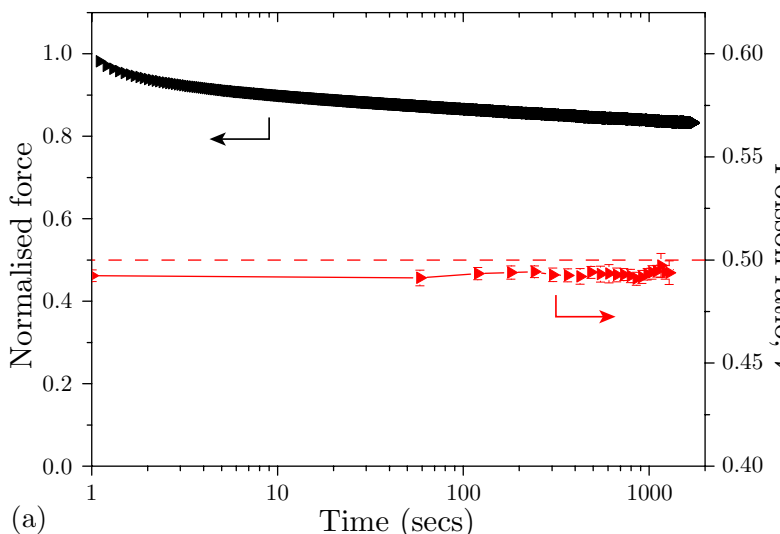

(a)

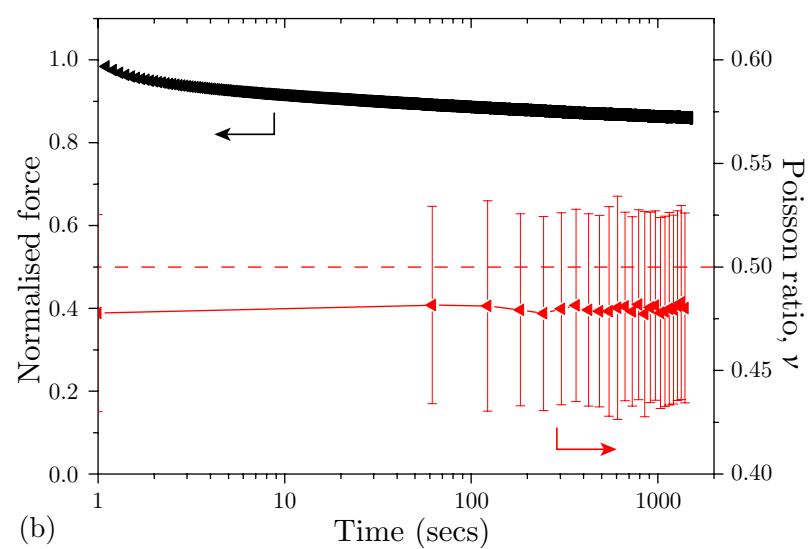

(b)

Figure 5: Normalised force and Poisson ratio relaxation of (a) a homogeneous and (b) an inhomogeneous carbon nanotube elastomer. Error bars show the standard deviation over the analysed region.

\subsection{Stress relaxation and (in) compressibility}

In this section, we present results utilising DIC to probe the time dependence of strain and ultimately the Poisson ratio. The Poisson ratio time dependence of an isotropic elastic material is an effect of its relation to the shear and bulk modulus:

$$
\nu=\frac{3 K-2 G}{2(3 K+G)},
$$

where $K$ and $G$ are the bulk modulus and shear modulus, respectively. In rubbers, the equilibrium bulk modulus is typically of the order 1-10 GPa whereas the shear modulus is $\sim 0.1-1 \mathrm{MPa}$ [15]. At lower $G / K$ Eq. 1 can be expanded to give $\nu=1 / 2+G / 3 K+\mathrm{O}(G / K)$; consequently, the Poisson ratio in rubbers is very close to 0.50 . Near the glass transition temperature and/or at short time scales, the polymer can undergo strain induced phase transition and enter the glassy regime; it should be noted that this transition is not discontinuous [30. In the glassy state, the shear modulus can increase by a factor of more than 1000 and becomes comparable to the bulk modulus [31]. Accordingly, the initial Poisson ratio will be at some glassy value of less than 0.50. Grassia et al. use a theoretical model to analyse results found in the literature, showing initial values of $0.35,0.40$, and 0.43 for polystyrene, polycarbonate, and a polycyanate, respectively, all of which relax to an equilibrium Poisson ratio of $\sim 0.50$ as the polymers revert to the rubbery regime. 32. The longest time scales of decay will be at the glass transition temperature, with decay times decreasing as temperature increases.

If time dependence is to be observed in a uniaxial step strain experiment, it is important that the applied strain occurs over a much shorter time scale than the shear modulus relaxation occurs. Furthermore, if the testing temperature is far from glass transition temperature strain induced glass transition may not be observable, the observable time scale being limited by the camera frame rate and the equipment strain rate. 


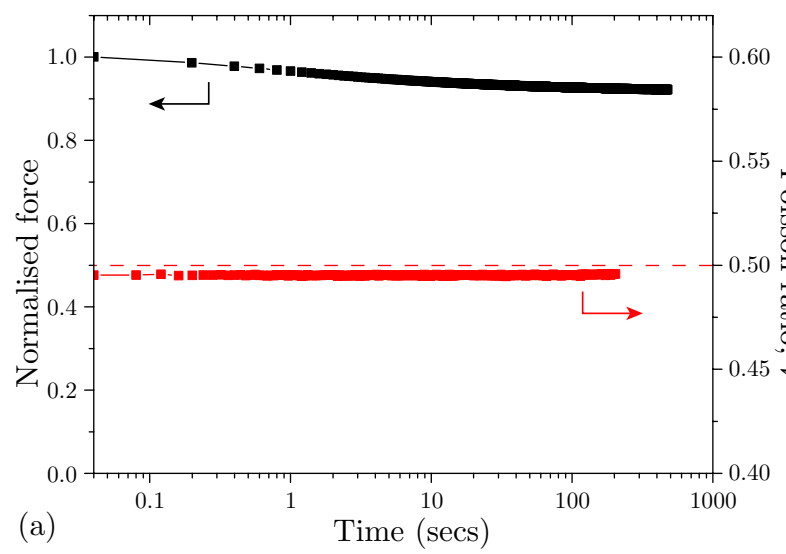

(a)

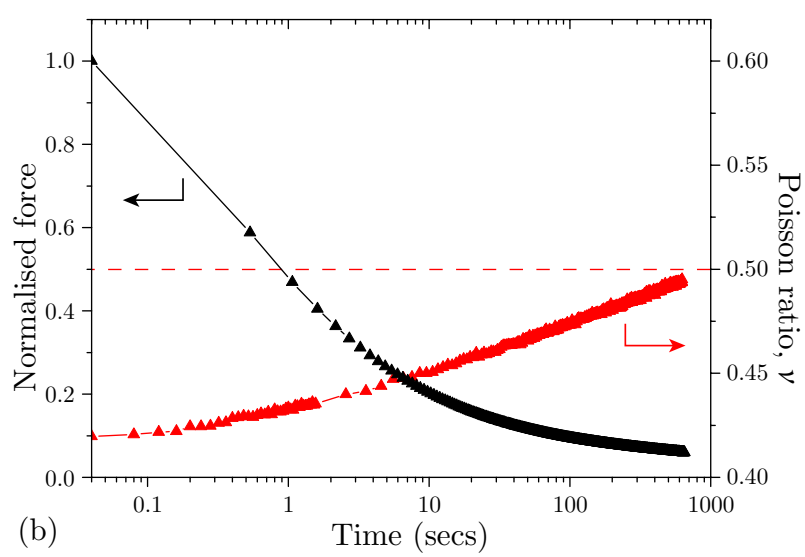

(b)

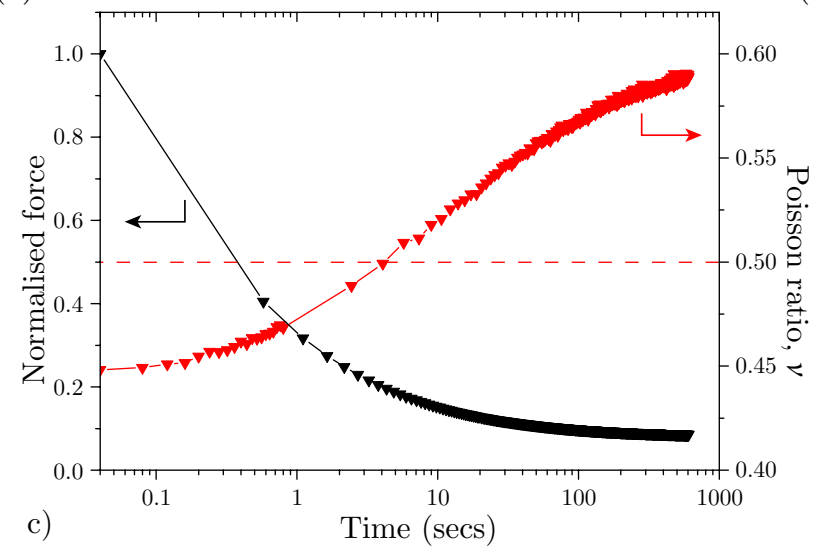

(c)

Figure 6: Normalised force and Poisson ratio relaxation after 10\% step strain for (a) polydimethylsiloxane, (b) polydomain liquid crystal elastomer, and (c) cholesteric liquid crystal elastomer

Figure 6 shows relaxation plots of normalised the force and Poisson ratio for three elastomers: PDMS, 3DLCE, and CLCE. PDMS was used as a control material as it is regularly used and its mechanical properties are well documented [33. The Poisson ratio is reported to be $0.50,1]$ but there few, if any, reports of the time dependent Poisson ratio. As seen in Fig 6 (a), the PDMS sample exhibits a small force relaxation and approaches an equilibrium value of roughly $90 \%$ the initial value. Over the probed time scales the Poisson ratio does not vary, deforming almost incompressibly with a value of 0.498 . Three repeats found values of $0.503,0.500$, and 0.499 . With the current experimental setup it is unlikely any time dependence would be observed as, at $-125{ }^{\circ} \mathrm{C}$, PDMS has one of lowest glass transition temperatures of all elastomers [34. As the Poisson ratio is a constant it can be inferred that the normalised force relaxation is identical to the normalised stress relaxation. It follows that due to the incompressibility, the stress relaxation is solely the result of the shear modulus' time dependence. Force relaxation and thus the shear modulus relaxation is roughly $10 \%$ and not of the order of magnitude difference needed to make it comparable to the bulk modulus.

Figure 6(b) shows the normalised force and Poisson ratio relaxation of a 3D-LCE. The force relaxation is substantial, converging to $0.8 \%$ the initial force value (not shown). Subsequently, the Poisson ratio also shows notable change, increasing from 0.42 and converging to 0.50 , the expected equilibrium value [15. The force relaxation in the CLCE (Fig 6 (c), is similar but settles to a slightly higher final value of $12 \%$ (not shown). Its Poisson ratio, starting at a value of 0.44 , approaches a value of 0.60 . This value suggests contraction of the CLCE, but it is infact due to the innate anisotropy of the CLCE, which causes a greater contraction ratio in $E_{x x}$ and a smaller contraction ratio out of plane. The convergent value is close to 0.62 
measured by Hirato (2008) 35] but less than the theoretically predicted value of 0.70. 15. Cuciuta et al. (2002) observed a value 0.72. However, in both experiments the Poisson ratio was not directly measured, rather it was interpreted from changes in the peak transmission wavelength. This reasoning is supported by Schmidtke et al., who demonstrated that under biaxial strain changes in peak transmission wavelength are linearly proportional to changes in elastomer thickness. The change in thickness was calculated from changes in light absorption of a dispersed dye [36. In future work on CLCEs, it would be useful to couple DIC measurements of in plane strains to out of plane measurements calculated from transmission wavelength shifts.

In Fig 7 we plot the bulk and shear modulus of the 3D-LCE as a function of time, where the relations $G=E / 2(1+\nu)$ and $K=E / 3(1-2 \nu)$ were used. $E$ is the Young's modulus and was calculated from the stress and the engineering strain, $\epsilon=\lambda_{\|}-1$, at time t. The stress was found from the force, $F(t)$, extracted from a spline fit to the force data, the initial area and $\lambda_{\perp}(t)$, such that $\sigma(t)=F(t) /\left(A_{0} \lambda_{\perp}(t)^{2}\right)$. Figure. 7 reveals that the shear modulus relaxation, and consequently a glass-rubber phase transition, can not possibly account for all the Poisson ratio relaxation. The shear modulus relaxes by a factor of just 20 , and by comparison to Fig 6(b), it is evident that the Poisson ratio is still increasing as the shear modulus begins to stabilise. The only alternative is to assume that the volume of the elastomer genuinely changes after an instant deformation, so that nano-voids open up in the network - and their gradual closure is what we observe during the $\nu(t)$ relaxation. This results in an effective lowering of $\mathrm{K}(\mathrm{t})$. There is some transition into the glass regime, which explains the bulk modulus (and shear modulus) relaxation at early times, but after 100 seconds the effect of nano-voids closing begins to take precedent and we see a rapid increase in $K(t)$ moving toward the GPa values of a typical rubber. Unfortunately, due to the error dependence on $1 /(1-2 \nu)$, as the 3D-LCE moves closer to 0.50 , the errors increase substantially and it is impossible to credibly determine the value of the Bulk modulus with high accuracy. The errors for the shear modulus are too small to be seen in the scale used but decrease as $1 /(1+\nu)$.

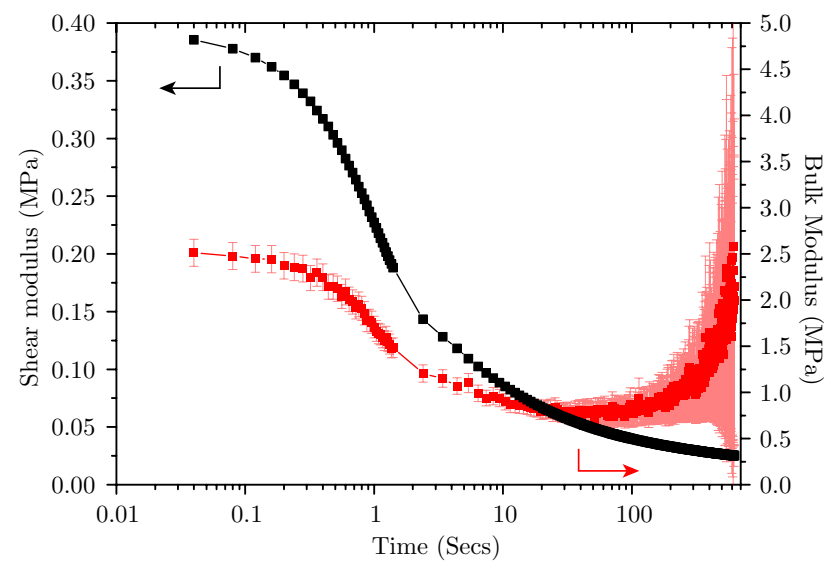

Figure 7: Plots of the shear and bulk modulus relaxation for 3D-LCE.

In Fig $8(a)$ the force and Poisson ratio relaxation plots of a fully swollen polyacrylamide (PAAm) gel uniaxially strained in water are shown. The origin of the Poisson ratio change in gels is not a glass phase transition, rather it is a result of their semi-open nature allowing them to exchange solvent and change their volume. This results in an effective lowering of $K(t)$, similar to the case of the 3D-LCE were volume change occurred due to the opening of nano-voids. It is known that free swollen gels will further take in solvent under extensive strain and expel water under compressive strain 18. The gel tested, of volume fraction 0.08, shows a smooth relation between force and Poisson ratio, reaching an equilibrium after 6 hours. The relaxation of force is attributed to a change in effective strain brought about by gel swelling, rather than viscoelastic effects. If the extensive force were removed from the gel, it would return to an unstrained state of larger volume than the initial state (due to solvent absorbtion), implying an effective 
reduction in imposed strain. The movement of solvent into the gel is a diffusion dominated process and as such depends on the dimensions of the gel [19]. Immediately after stretch, the Poisson ratio is 0.50 and the gel has deformed incompressibly as there has been little time for solvent exchange. As the gel swells, the Poisson ratio decreases, as does force, and after 6 hours equilibrates to a value of 0.26 . This is in good agreement with the 0.25 predicted by thermodynamic theories of strain induced swelling for dilute neutral gels [18. Figure 8(b) shows the shear and bulk modulus relaxation. Similar to the case of the 3D-LCE, the Poisson ratio change is primarily in response to volume change resulting in an effective lowing of $K(t)$. The shear modulus shows little change with time and remains at approximately $16 \mathrm{KPa}$.

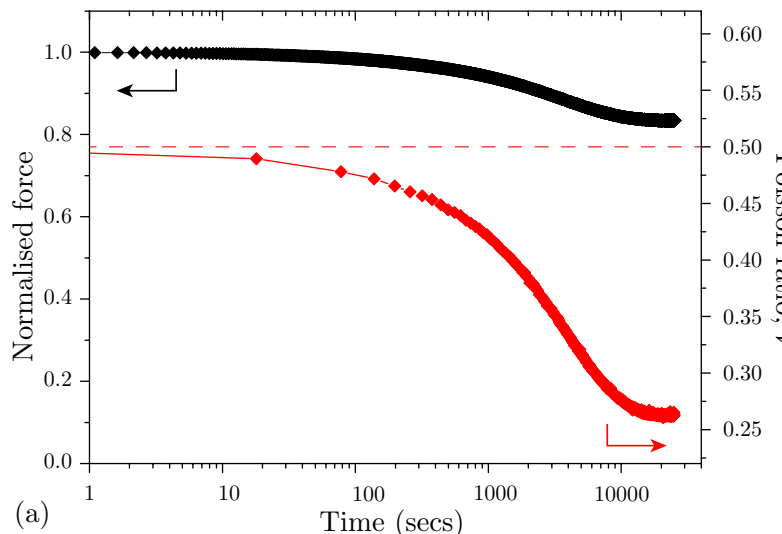

(a)

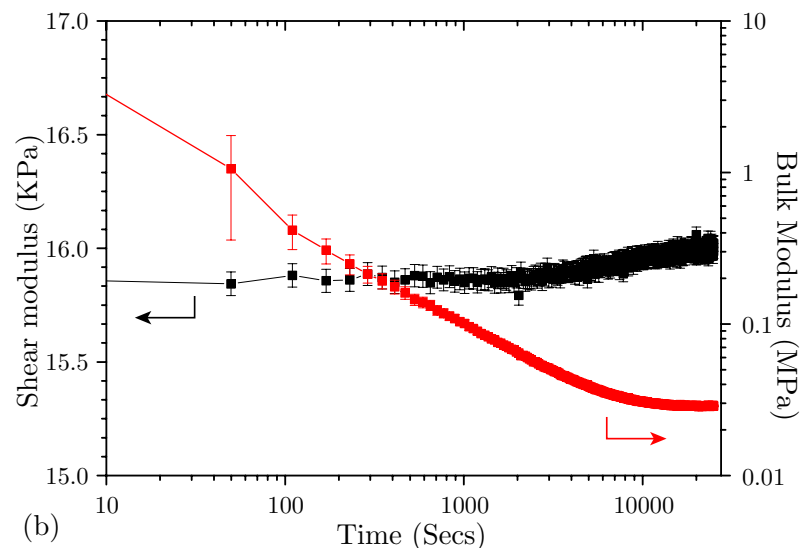

(b)

Figure 8: (a) Normalised force and Poisson ratio relaxation of a polyacrylamide gel immersed in water in response to a $10 \%$ step strain. (b) Corresponding shear and bulk moduli.

\section{Discussion}

Using DIC on soft materials is a powerful technique but as with any experimental technique certain conditions must be met in order to achieve accurate results. With soft materials, there can be difficulty ensuring they deform in plane and that they are flat before stretch. Problems can arise with the material deforming under its own weight or defects in the manufacturing process can result in a preferentially curved surface. This can be a major problem in $2 \mathrm{D}$ digital image correlation but can be resolved by using $3 \mathrm{D}$ digital image correlation, which uses two cameras to allow computation of strains in the out of plane direction. Of particular importance when conducting DIC is the choice of subset size and strain window size, which determine the spatial resolution of displacement and strain fields, respectively. The subset size determines the number of pixels in each localised region in the undeformed image to be mapped to the deformed image. If this subset is too small, the correlation between images may not be good enough to give reliable results, and if it is too big, it may mask inhomogeneous deformation as well as increasing computation time. For homogeneous tests, it is better to consider a larger subset which will substantially increase the resolution of displacement results (as is the case for strain fields). For heterogeneity, of course, and high spatial frequencies, the smallest subset possible should be chosen in order to capture this. The subset and strain windows actually act as low-pass filters in the displacement and strain field regimes, filtering out high spatial frequencies which typically arise from noise. In conclusion, the choice of parameters depends on the aim of the measurement.

Strains in DIC typically undergo some level of smoothing to increase their accuracy. In this analysis smoothing is implemented through a spline fit over displacement points inside strain windows, strains are subsequently calculated through the gradient. A large strain window can increase the accuracy of the calculated strains but it may also mask inhomogeneities [5]. This is demonstrated in Fig. 9] with plot a, b, and c showing Poisson ratio maps of PDMS calculated from strain windows of 15, 30, and 60 displacement 
points respectively. The DIC parameters are shown in table. 1, where the resolutions are listed from 30 displacement points. With increasing subset size the plots become smoother and appear more homogeneous, this is particular noticeable around the clamp effects. This is due to the spatial resolution decreasing from 1.6 $\mathrm{mm}$, to $2.9 \mathrm{~mm}$ to $5.3 \mathrm{~mm}$ and the SVG resolution increasing from $601 \mu$ strain to $200 \mu$ strain to $61 \mu$ strain. The average Poisson ratio, calculated over the homogeneous region denoted by dashed box, is equal to 0.498 for all strain windows used. However, for larger strain windows, this begins to decrease as the windows encroach on the clamped region. Strain window size is not important when calculated average values for a system, which is the typically reported quantity for Poisson ration, but will affect the smoothness of strain maps. For samples which are typically homogeneous it is advised that larger strain windows are used to increase local accuracy. In the case of inhomogeneous samples, smaller strain windows are recommended so as to not mask information. [5] For average values, the strain window size is not as important.

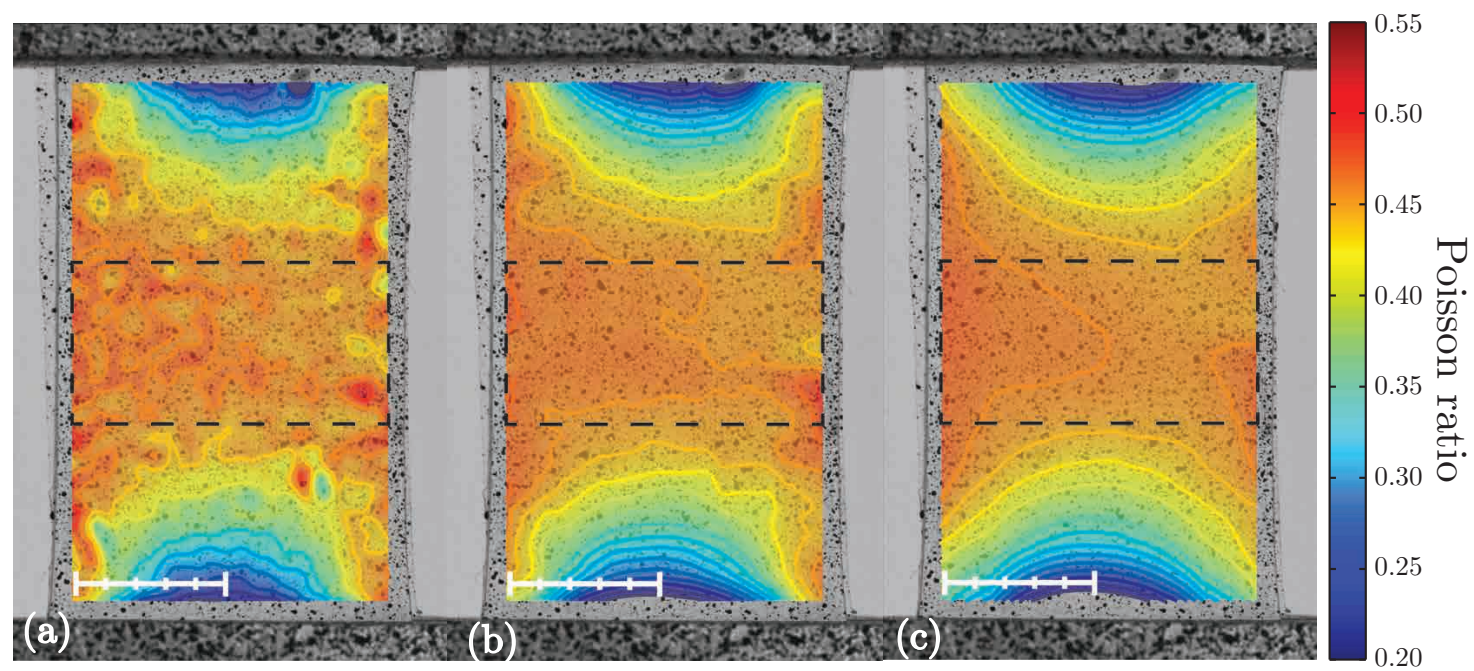

Figure 9: Poisson ratio maps in PDMS calculated from a strain windows size of (a) 15, (b) 30, and (c) 60 displacement points. The dashed black rectangle indicates the region of interest in which an average is taken to give the Poisson ratio of PDMS. The scale bar represents $5 \mathrm{~mm}$.

The average Poisson ratio for a material is taken over a user selected region of interest (ROI). For homogeneous materials this region must be selected over the area that deformed homogeneously, e.g., away from the clamps. Depending on where the ROI is placed, there can be small changes in the Poisson ratio, so it is important to take the average over a uniformly deformed area as large as possible if the material response is to be truly represented. For inhomogeneous materials, the ROI will have a more dramatic influence on Poisson ratio, and it becomes essential to have as large a ROI as possible, preferably covering the whole material surface.

Research cameras can be expensive and with their relative inexpensiveness and improved quality, DSLR cameras are becoming a viable alternative. Most DSLRs have mechanical shutters and for long time experiments this may be a problem as it can cause the camera to move slightly over time, creating artificial time dependent strains. It is important to place the camera in a secure (preferable full body supporting) mount and allowing the camera to fire a few hundred times to settle (which can be automated). Images taken on a rigid body can then be used to test for movement. For video footage there is no mechanical action and there is no issue. The use of DSLRs is not advised for small strains ( $10 \mu$ strains) as their performance is not yet on par with research cameras. However, as can be seen from the resolutions in table. 1, their performance is adequate for the large strains typically applied to elastomeric materials.

In summary, we have demonstrated the application of DIC for soft materials in a large range of temporal and spatial resolutions. This was demonstrated with uniaxial strain and relaxation experiments on PDMS, homogeneous and inhomogeneous CNT, 3D-LCE, CLCE, and a PAAm gel immersed in a good solvent. 
PDMS was used as a control and the results were found to agree precisely with those found in the literature. The differences between inhomogeneous and homogeneous CNT elastomers were easily observable, and their average Poisson ratios were shown to be dominated by the polymer response, leading to an almost incompressible deformation. Liquid crystal elastomers were demonstrated to have a time dependent Poisson ratio, with the 3D-LCE converging to an incompressible value of 0.5 and the anisotropic CLCE converging to 0.60 . We find the Poisson relaxation in the 3D-LCE could only be explained with a genuine change in volume brought about by the opening, and subsequent closing, of nano-voids, resulting in an effective lowering in $K(t)$. Finally, the DIC was shown to give good results for a PAAm gel immersed water, which initially deformed incompressibly and relaxed to a Poisson ratio 0.26 , in good agreement with theory. In PAAm, the Poisson ratio relaxation is entirely attributed to volume change and an effective lowering of $K$.

\section{Acknowledgements}

This work was supported by EPSRC UK. The authors are grateful to Dr J.E. Marshall and Y.Y.S. Huang for advice and useful discussions.

[1] J. E. Mark, Polymer Data Handbook (2nd Edition), Oxford University Press, 2009.

[2] D. Tscharnuter, M. Jerabek, Z. Major, R. W. Lang, Time-dependent poisson's ratio of polypropylene compounds for various strain histories, Mech. Time-Depend. Mater. 15 (2011) 15-28.

[3] N. W. Tschoegl, W. G. Knauss, I. Emri, Poisson's ratio in linear viscoelasticity - a critical review, Mech. Time-Depend. Mater. 6 (2002) 3-51.

[4] A. J. W. McClung, G. P. Tandon, K. E. Goecke, J. W. Baur, Non-contact technique for characterizing full-field surface deformation of shape memory polymers at elevated and room temperatures, Polym. Test. 30 (2011) 140 - 149.

[5] P. Lava, S. Cooreman, D. Debruyne, Study of systematic errors in strain fields obtained via dic using heterogeneous deformation generated by plastic fea, Opt. Laser Eng. 48 (2010) $457-468$.

[6] W. H. Peters, W. F. Ranson, Digital image techniques in experimental stress analysis, Optical Engineering 21 (1982) $427-432$

[7] H. Schreirer, J. J. Orteu, M. Sutton, Image correlation for shape, motion and deformation measurements, Springer US, 2009.

[8] P. Reu, Introduction to digital image correlation: Best practices and applications, Exp Techniques 36 (2012) 3-4.

[9] H. Bechir, L. Chevalier, M. Chaouche, K. Boufala, Hyperelastic constitutive model for rubber-like materials based on the first seth strain measures invariant, Eur. J. Mech. A-solid 25 (2006) $110-124$.

[10] S. Lee, F. Barthelat, N. Moldovan, H. D. Espinosa, N. G. Wadley, Deformation rate effects on failure modes of open-cell al foams and textile cellular materials, Int J Solids Struct 2006 (2006) 53-73.

[11] A. Willems, S. Lomov, I. Verpoest et al., Drape-ability characterisation of textile composite reinforcemetns using digital image correlation, Opt. Laser Eng. 47 (2009) 343-351.

[12] T. Cheng, C. Dai, R. Gan, Viscoelastic properties of human tympanic membrane, Ann. Biomed. Eng. 35 (2007) $305-314$.

[13] J. Ning, V. G. Braxton, Y. Wang, M. A. Sutton, Y. Wang, S. M. Lessner, Speckle patterning of soft tissues for strain field measurement using digital image xorrelation: Preliminary quality assessment of patterns, Microsc. Microanal. 17 (2011) $81-90$.

[14] Y. Y. Huang, S. V. Ahir, E. M. Terentjev, Dispersion rheology of carbon nanotubes in a polymer matrix, Phys. Rev. B 73 (2006) 125422.

[15] E. M. Terentjev, M. Warner, Liquid crystal elastomers, Oxford University Press, 2007.

[16] A. Hotta, E. M. Terentjev, Long-time stress relaxation in polyacrylate nematic liquid crystalline elastomers, J. Phys.: Cond. Matter 13 (2001) 11453-11464.

[17] P. Cicuta, A. Tajbakhsh, E. M. Terentjev, Evolution of photonic structure on deformation of cholesteric elastomers, Phys. Rev. E 65 (2002) 1-6.

[18] K. Urayama, T. Takigawa, Volume of polymer gels coupled to deformation, Soft Matter 8 (2012) 8017-8029.

[19] A. Konda, K. Urayama, T. Takigawa, Strain-rate-dependent poisson's ratio and stress of polymer gels in solvents revealed by ultraslow stretching, Macromolecules 44 (2011) 3000-3006.

[20] H. Hencky, Über die form des elastizitatsgesetzes bei ideal elastischen stoffen, Z. Techn. Phys. 9 (1928) 214

[21] Y. Ji, Y. Y. Huang, A. R. Tajbakhsh, E. M. Terentjev, Polysiloxane surfactants for the dispersion of carbon nanotubes in nonpolar organic solvents, Langmuir 25 (2009) 12325-12331.

[22] J. Küpfer, H. Finkelmann, Nematic liquid single crystal elastomers, Macromol. Chem. Rapid Commun. 12 (1991) 717-726.

[23] A. Hotta, E. M. Terentjev, Dynamic soft elasticity in monodomain nematic elastomers, Eur. Phys. J. E 10 (2003) $291-301$.

[24] S. M. Clarke, E. M. Terentjev, Slow stress relaxation in randomly disordered nematic elastomers and gels, Phys. Rev. Lett. 81 (1998) 4436-4439.

[25] S. T. Kim, H. Finkelmann, Cholesteric liquid single-crystal elastomers (lsce) obtained by the anisotropic deswelling method, Macromol. Chem. Rapid Commun. 22 (2001) 429-433. 
[26] M. A. Sutton, J. J. Orteu, H. Schreier, Image correlation for shape, motion and deformation measurements, Springer Berlin Heidelberg, 2009.

[27] J. Lu, Elastic properties of single and multilayered nanotubes, J. Phys. Chem. Solids 58 (1997) $1649-1652$.

[28] J. Coleman, U. Khan, W. Blau et al., Small but strong: A review of the mechanical properties of carbon nanotube-polymer composites, Carbon 44 (2006) 1624-1652.

[29] M. A. Sutton, J. H. Yan, V. Tiwari, H. W. Schreier, J. J. Orteu, The effect of out-of-plane motion on 2d and 3d digital image correlation measurements, Opt. Laser Eng. 46 (2008) $746-757$.

[30] G. N. Greaves, A. L. Greer, R. S. Lakes, T. Rouxel, Poisson's ratio and modern materials, Nature Mater. 10 (2011) 823-838.

[31] R. S. Lakes, A. Wineman, On poisson's ratio of linearly viscoelastic solids, J Elasticity 85 (2006) 45-63.

[32] L. Grassia, A. D'Amore, S. L. Simon, On the viscoelastic poisson's ratio in amorphous polymers, J. Rheol. 54 (2010) 1009-1022.

[33] A. Mata, A. Fleischman, S. Roy, Characterization of polydimethylsiloxane (pdms) properties for biomedical micro/nanosystems., Biomed 7 (2005) 281-293.

[34] F. Schnieder, T. Fellner, J. Wilde et al., Mechanical properties of silicone for mems, J. Micromech. Microeng. 18 (2008) 065008.

[35] Y. Hirota, Y. Ji, F. Serra, A. R. Tajbakhsh, E. M. Terentjev, Effect of crosslinking on the photonic bandgap in deformable cholesteric elastomers, Opt. Express 16 (2008) 5320-5331.

[36] J. Schmidtke, S. Kniesel, H. Finkelmann, Probing the photonic properties of a cholesteric elastomer under biaxial stress, Macromolecules 38 (2005) 1357-1363. 